\title{
Addition energies and density dipole response of quantum rings under the influence of in-plane electric fields
}

\author{
Francesc Malet, Martí Pi, and Manuel Barranco \\ Departament ECM and IN ${ }^{2}$ UB, Facultat de Física, Universitat de Barcelona, Diagonal 647, 08028 Barcelona, Spain
}

Llorenç Serra

Departament de Física, Universitat de les Illes Balears and Institut de Física Interdisciplinària i de Sistemes Complexos IFISC (CSIC-UIB), E-07122 Palma de Mallorca, Spain

Enrico Lipparini

Dipartimento di Fisica, Università di Trento and INFN, 38050 Povo (Trento), Italy

(Received 12 July 2007; published 3 December 2007)

\begin{abstract}
Within density functional theory, we address the effect of an in-plane electric field $\mathcal{E}$ on the ground state and the density dipole response of a many-electron quantum ring, which is also submitted to a perpendicular magnetic field $B$. Addition energies and density dipole spectra are discussed as a function of $\mathcal{E}$. For the two-electron case, an exact numerical calculation is performed, obtaining the spin-phase diagram in the $\mathcal{E}$ - $B$ plane and showing that transitions between singlet and triplet spin states can be induced by varying the fields. We also find that, in spite of the deformation that $\mathcal{E}$ causes in the electronic density, the spin of the ground state of a given electron number ring is very robust, changing little as the strength of the electric field is reasonably increased.
\end{abstract}

DOI: 10.1103/PhysRevB.76.245302

\section{INTRODUCTION}

Spin effects in two-dimensional (2D) nanostructures have been widely investigated in recent years because of their potential relevance to quantum information processing schemes. Experimental and theoretical results involving, e.g., spin pairing, ${ }^{1,2}$ Zeeman splitting ${ }^{3-6}$ and spin states ${ }^{7,8}$ due to in-plane or perpendicular magnetic fields, spin blockade effects, ${ }^{8-10}$ and, of particular interest, spin transitions driven by magnetic fields ${ }^{11-19}$ or gate voltage ${ }^{20-23}$ have been reported.

The possibility to externally control and induce transitions between entangled spin-singlet $|\uparrow \downarrow\rangle-|\downarrow \uparrow\rangle$ and nonentangled spin-triplet $|\uparrow \uparrow\rangle$ states is of great importance in the realization of qubits since it provides an efficient way to manipulate the entanglement of such systems. Coulomb and spin blockade spectroscopy experiments with lateral ${ }^{16,21}$ and vertical $^{13,19}$ quantum dot (QD) systems, in which the QD's are formed by confining a few electrons of a $2 \mathrm{D}$ electron gas by the application of gate voltages, have proved that the spin of the ground state (gs) can be manipulated by magnetic fields $(B),{ }^{16}$ or by gate voltages at fixed $B,{ }^{21}$ the latter being a more efficient procedure since the gate voltage can, in general, be tuned faster than the magnetic field. ${ }^{24}$ Similar conclusions have been obtained from experiments with manyelectron quantum rings (QRs) fabricated with scanning force microscope nanolithographic techniques on an AlGaAsGaAs heterostructure. ${ }^{22,23}$ In these ring structures, the effect of both magnetic and electric $(\mathcal{E})$ fields on the gs spin has been investigated.

Motivated by these experimental results, we have addressed, within local spin-density functional theory (LSDFT), the structure of GaAs quantum rings hosting up to $N=12$ electrons as a function of $B$ and $\mathcal{E}$ and have used these results to obtain their addition energies and density dipole
PACS number(s): 73.21.-b, 73.22.-f, 71.15.Mb

response for some selected configurations. We have singled out the $N=2$ electron case and have solved it exactly, obtaining its spin-phase diagram in the $\mathcal{E}$ - $B$ plane and showing that, indeed, spin transitions can be externally induced at fixed magnetic field by varying the electric field, and vice versa.

The paper is organized as follows. In Sec. II, we briefly introduce the LSDFT and show the gs results as a function of $B$ and $\mathcal{E}$. In Sec. III, we describe the time-dependent LSDFT (TDLSDFT) approach that we have used to calculate the density dipole response and the method employed to obtain the excitation frequencies of the system. A sum rule approach to discuss the electric dipole polarizability is also presented. The exact results for $N=2$ are presented and discussed in Sec. IV and, finally, a summary is drawn in Sec. V.

\section{DENSITY FUNCTIONAL CALCULATION FOR THE GROUND STATE OF THE MANY-ELECTRON QUANTUM RING}

We consider a system of $N$ electrons, whose motion is restricted to the $x y$ plane, where a suitable confining potential $V_{\text {conf }}(r)$, with $r=\sqrt{x^{2}+y^{2}}$, induces the formation of a ringshaped electron island. The system is placed in a magnetic field perpendicular to the plane where the electrons move, and an electric field $\mathcal{E}$ is applied in the $x$ direction. Both fields are static and uniform. In the effective mass, dielectric constant approximation, the single-electron wave functions are given by the solution of the Kohn-Sham (KS) equations

$$
\begin{aligned}
\left\{\frac{1}{2 m} \mathbf{P}^{2}+\frac{e}{\epsilon} \mathcal{E} x+V_{\text {conf }}(r)+V^{H}+V^{x c}\right. \\
\left.+\left[W^{x c}+\frac{1}{2} g^{*} \mu_{B} B\right] \sigma_{z}\right\} \varphi_{\alpha}(\mathbf{r})=\epsilon_{\alpha} \varphi_{\alpha}(\mathbf{r}),
\end{aligned}
$$

where $m=m^{*} m_{e}$ is the electron effective mass in units of the 


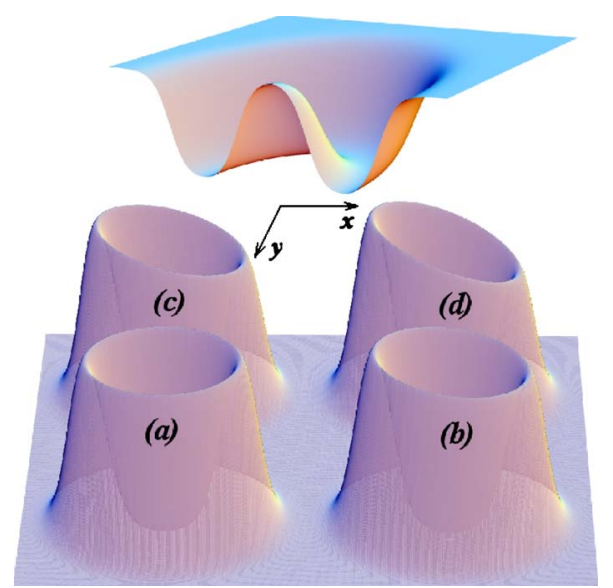

FIG. 1. (Color online) Confining potential $V_{\text {conf }}(r)$ (upper) and electronic densities (lower) for the $N=10$ ring at $B=0$ and $\mathcal{E}=0,1$, 2 , and $3 \mathrm{mV} / \mathrm{nm}$, (a)-(d) configurations, respectively.

bare electron mass $m_{e}, \mu_{B}=\hbar e /\left(2 m_{e} c\right)$ is the Bohr magneton, $g^{*}$ and $\epsilon$ are, respectively, the effective gyromagnetic factor and the dielectric constant, $\sigma_{z}$ is the $z$ component of the Pauli spin-vector operator $\sigma$, and $\mathbf{P}=-i \hbar \nabla+e \mathbf{A} / c$ represents the canonical momentum in terms of the vector potential $\mathbf{A}$, which we write in the symmetric gauge, $\mathbf{A}=B(-y, x, 0) / 2$, with $\mathbf{B}=\nabla \times \mathbf{A}=B \hat{\mathbf{z}}$. In the above equation, $V^{H}$ is the Hartree potential, and $V^{x c}=\partial \mathcal{E}_{x c}(n, m) /\left.\partial n\right|_{g s}$ and $W^{x c}$ $=\partial \mathcal{E}_{x c}(n, m) /\left.\partial m\right|_{g s}$ are the variations of the exchangecorrelation energy density $\mathcal{E}_{x c}(n, m)$ in terms of the local electronic density $n=n^{\uparrow}+n^{\downarrow}$ and spin magnetization $m=n^{\uparrow}$ $-n^{\downarrow}$ taken at the gs, where $n^{\uparrow}$ and $n^{\downarrow}$ are, respectively, the spin up and down local electron densities. The exchangecorrelation energy density $\mathcal{E}_{x c}$ has been constructed from the results of the nonpolarized and fully polarized 2D electron gas, ${ }^{25}$ using the two-dimensional von Barth and Hedin ${ }^{26}$ prescription to interpolate between both regimes.

To simplify the expressions, in the following, we shall mostly use effective atomic units $\hbar=e^{2} / \epsilon=m=1$, for which the length unit is the Bohr radius $a_{0}$ times $\epsilon / \mathrm{m}^{*}$ and the energy unit is the Hartree times $m^{*} / \epsilon^{2}$, which we call, respectively, $a_{0}^{*}$ and $E_{H}^{*}$. For the GaAs, $g^{*}=-0.44, m^{*}=0.067$, and $\epsilon=12.4$. Thus, $a_{0}^{*} \simeq 97.9 \AA$ and $E_{H}^{*} \simeq 11.9 \mathrm{meV}$.

The confining potential $V_{\text {conf }}(r)$ modeling the ring has been chosen of the following smooth form

$$
V_{\text {conf }}(r)=V_{0}^{(1)} \frac{1}{1+e^{\left(r-R_{0}+w\right) / \gamma_{1}}}+V_{0}^{(2)} \frac{e^{\left(r-R_{0}-w\right) / \gamma_{2}}}{1+e^{\left(r-R_{0}-w\right) / \gamma_{2}}},
$$

where $R_{0}=2.5, V_{0}^{(1)}=V_{0}^{(2)}=5, \gamma_{1}=\gamma_{2}=0.3$, and $w=1.25$ in effective atomic units. This corresponds to a fairly thick ring of average radius $R_{0} \sim 25 \mathrm{~nm}$ and $2 w \sim 25 \mathrm{~nm}$ width. A plot of $V_{\text {conf }}$ is shown in Fig. 1.

In the presence of an electric field, the system is no longer circularly symmetric and the KS equations are 2D partial differential equations, which we have solved in Cartesian coordinates after having discretized the differential operators using 11 point formulas and employed fast-Fourier transform techniques ${ }^{27}$ to obtain the Hartree potential. The essential

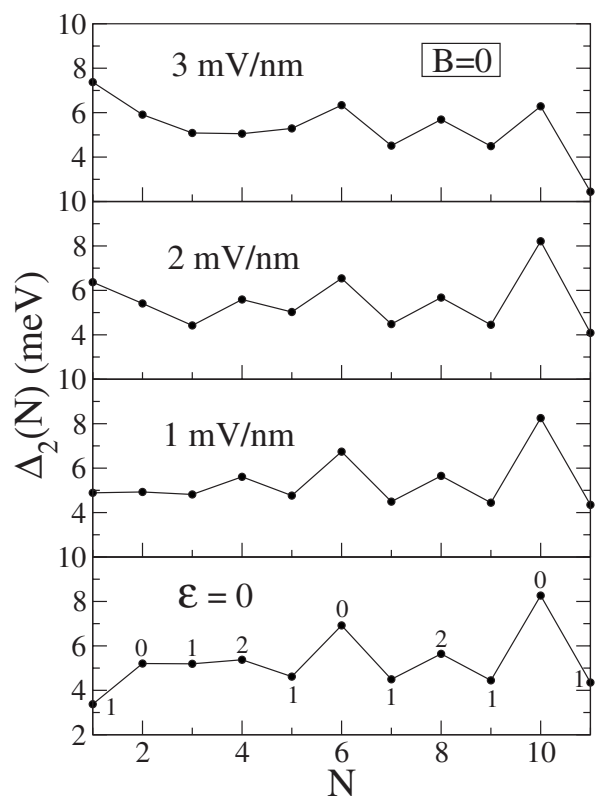

FIG. 2. Addition energies for $B=0$ and $\mathcal{E}=0,1,2$, and $3 \mathrm{mV} / \mathrm{nm}$. The value of $2 S_{z}$ is indicated in the $\mathcal{E}=0$ panel.

details of our method are given in Ref. 28. In the lower panels of Fig. 1, we plot the ground state density of the $N$ $=10$ ring at $B=0$ and $\mathcal{E}=0-3 \mathrm{mV} / \mathrm{nm}$, which shows how the electronic density is progressively deformed along the direction of the applied electric field as the value of $\mathcal{E}$ is increased. In spite of the progressive deformation of the quantum ring as $\mathcal{E}$ increases, all the electronic configurations we have found in our study are always smooth, without chargedensity nor spin-density waves in the azimuthal direction. We attribute this to the external ring potential we have used, which is fairly wide radially, as we have already mentioned. For more quasiunidimensional rings and/or electronic systems more dilute than ours, sometimes the LSDFT yields solutions presenting azimuthally modulated charge and spin densities. We refer the reader to Ref. 29 for a detailed discussion of these particular solutions arising in LSDFT.

Figures 2-4 show the addition energies $\Delta_{2}(N)=E(N+1)$ $-2 E(N)+E(N-1)$ for quantum rings containing up to $N$ $=12$ electrons and for some selected values of the electric and magnetic fields. From the maxima in $\Delta_{2}(N)$, at $\mathcal{E}=0$ and $B=0$, one can identify shell closures at $N=6$ and 10 , as found in previous calculations for parabolically confined QRs. ${ }^{30,31}$ As $\mathcal{E}$ starts increasing, the ring becomes more and more deformed, leading to a shell structure in which the sequence of magic numbers differs from the circular case. This can be seen, for example, for $\mathcal{E}=1 \mathrm{mV} / \mathrm{nm}$ and $B=0$, where a small peak arises at $N=4$. Increasing further the electric field while switching on the magnetic field, the addition spectrum displays less structure. The peak at $N=8$ is the sole exception, indicating a very strong shell closure for the chosen $V_{\text {conf }}$.

The ground state spin, $S(N)$, is also indicated in the figures. It can be seen that, for the displayed $N$ values, at $B$ $=0$, the spin is unaffected by electric fields up to $\mathcal{E}$ $=3 \mathrm{mV} / \mathrm{nm} . S(N)$ corresponding to odd $-N$ rings turns out to be very robust even when a magnetic field is applied and 


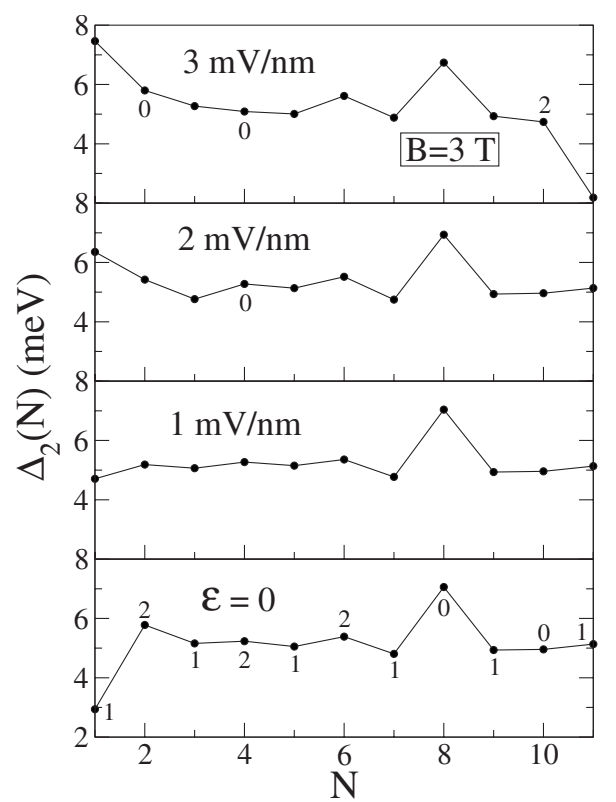

FIG. 3. Same as Fig. 2 for $B=3$ T. Upper panels display the value of $2 S_{z}$ only when it differs from that of the $\mathcal{E}=0$ case.

does not change for any of the values of $\mathcal{E}$ and $B$ that we have considered. The same occurs for the $N=8$ case. However, at $B \neq 0$, interesting features appear for the rest of even electron numbers, namely, $N=2,4,6$, and 10 . Indeed, one can observe transitions between $2 S(N)=0$ and $2 S(N)=2$ states driven by the electric field at fixed $B$. Similar spin transitions induced by an applied magnetic field have been observed in Coulomb blockade spectroscopy experiments with vertical and lateral few-electron quantum dots ${ }^{16}$ or by the application of a gate voltage at fixed $B .{ }^{21}$ This technique has also been used in experiments with many-electron quantum rings, ${ }^{22,23}$ where the gate voltage-induced singlet-triplet

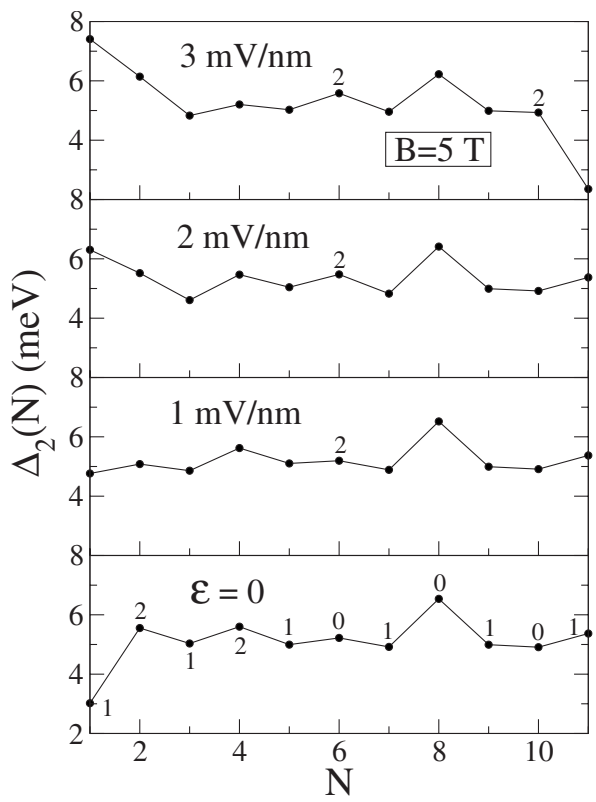

FIG. 4. Same as in Fig. 3 for $B=5$ T.

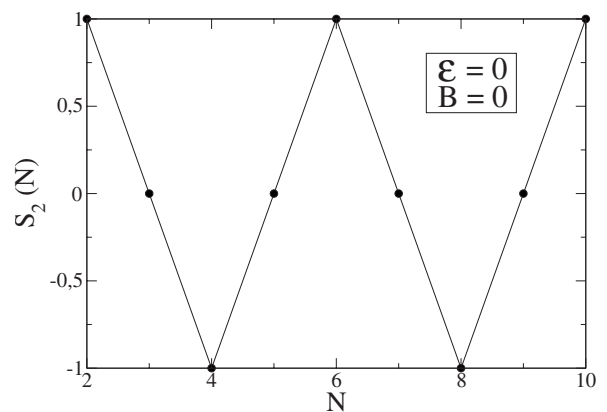

FIG. 5. Second spin differences $S_{2}(N)$ for $B=0 \mathrm{~T}$ and $\mathcal{E}$ $=0 \mathrm{mV} / \mathrm{nm}$.

transitions have been related to the competition between the Hartree and the exchange interactions, which favor, respectively, the formation of singlet and triplet spin states. The second spin differences, $S_{2}(N)=S(N+1)-2 S(N)+S(N-1)$, have also been measured from the slopes of the Coulomb blockade peak spacings. ${ }^{23}$ Our results at $\mathcal{E}=B=0$ are shown in Fig. 5. It can be seen that $S_{2}(N)$ takes the three integer values $-1,0$ and 1 , with one-unit jumps. The experimental $N$ sequence matches that of our calculation except in one case, in which it passes directly from the value 1 to -1 . We have found these two-unit jumps only when $B \neq 0$.

\section{DENSITY DIPOLE RESPONSE}

\section{A. Electric dipole polarizability and sum rules}

Some global properties of the quantum ring excitation spectrum can be obtained by using sum rule techniques. For a general excitation operator $F$, one can define the strength function

$$
S(\omega)=\sum_{j}\left|\left\langle\Psi_{0}|F| \Psi_{j}\right\rangle\right|^{2} \delta\left(\omega-\omega_{j 0}\right),
$$

where $\left|\Psi_{j}\right\rangle$ and $\omega_{j 0}$ are, respectively, the $j$ th excited state and its excitation energy, and $\left|\Psi_{0}\right\rangle$ is the ground state of the many-electron Hamiltonian

$$
\begin{aligned}
H= & \frac{1}{2 m} \sum_{i=1}^{N}\left[\mathbf{P}^{2}+V_{\text {conf }}(r)+\frac{e}{\epsilon} \mathcal{E} x+\frac{1}{2} g^{*} \mu_{B} B \sigma_{z}\right]_{i} \\
& +\sum_{i<j=1}^{N} \frac{e^{2}}{\epsilon\left|\mathbf{r}_{i}-\mathbf{r}_{j}\right|} .
\end{aligned}
$$

The sum-or integral in the case of continuum spectrum-in Eq. (3) extends over all the excited states of the system, and the energy moments of the strength function are given by

$$
m_{k}=\int_{0}^{\infty} d \omega \omega^{k} S(\omega)=\sum_{j} \omega_{j 0}^{k}\left|\left\langle\Psi_{0}|F| \Psi_{j}\right\rangle\right|^{2},
$$

which define the so-called sum rules. ${ }^{32-34}$ Of particular interest are the $m_{-1}, m_{1}$, and $m_{3}$ moments. For the dipole operator along the $x$ direction, $F=D^{x}=e \sum_{i=1}^{N} x_{i}$, they read

$$
m_{-1}=\frac{1}{2} \alpha
$$


TABLE I. Electric dipole polarizability in effective atomic units for rings with different electron numbers as a function of the magnetic field $B$.

\begin{tabular}{ccccc}
\hline \hline $\begin{array}{c}B \\
(\mathrm{~T})\end{array}$ & $N=6$ & $N=8$ & $N=10$ & $N=12$ \\
\hline 0 & 17.34 & 17.82 & 17.96 & 18.99 \\
3 & 18.20 & 17.77 & 18.59 & 19.25 \\
5 & 18.51 & 18.28 & 19.18 & 19.83 \\
\hline \hline
\end{tabular}

$$
\begin{gathered}
m_{1}=\frac{1}{2}\left\langle 0\left|\left[D^{x},\left[H, D^{x}\right]\right]\right| 0\right\rangle=\frac{N}{2}, \\
m_{3}=\frac{1}{2}\left\langle 0\left|\left[\left[D^{x}, H\right],\left[H,\left[H, D^{x}\right]\right]\right]\right| 0\right\rangle \\
=\frac{N}{2} \omega_{c}^{2}+\frac{1}{2} \int d \mathbf{r} V_{\text {conf }}(r)\left[\nabla_{x}^{2} n(\mathbf{r})\right],
\end{gathered}
$$

where $\omega_{c}=e B /(m c)$ is the cyclotron frequency. Since the ground state of the ring is deformed when $\mathcal{E} \neq 0$, excitations caused by the dipole operator in the $x$ and $y$ directions, $D^{x}$ $=e \sum_{i=1}^{N} x_{i}$ and $D^{y}=e \sum_{i=1}^{N} y_{i}$, are not the same. $\alpha$ is the electric dipole polarizability, which can be obtained as

$$
\alpha=\left.\frac{d^{2}\langle H\rangle}{d \mu^{2}}\right|_{\mu=0},
$$

with $\mu \equiv e \mathcal{E} / \epsilon . m_{1}$ is just the Thomas-Reiche-Kuhn sum rule, ${ }^{35}$ and $m_{3}$ can be easily calculated from the ground state density $n(\mathbf{r})$. The $m_{3}$ sum rule for $D^{y}$ involves the $\nabla_{y}^{2}$ derivative.

The electric polarizabilty $\alpha$ is a measurable quantity for many systems, such as atoms and nuclei. Its determination requires an accurate evaluation of the total energy of the deformed system as a function of $\mathcal{E}$ in the limit of zero applied electric field. We give in Table I the LSDFT values of the polarizability in effective atomic units for some selected values of $N$ as a function of the magnetic field. One can see that $\alpha$ turns out to be rather insensitive to $B$ and $N$. We recall that the dipole polarizability is a length to the cube, and the only length playing a role here is the mean radius $R_{0}$ of the confining potential, whose cube (15.63) is about 20\%-30\% smaller than the value of the polarizability. The enhancement of $\alpha$ with respect to $R_{0}^{3}$ is a measure of the quantum spillout of the electrons outside the "ideal" ring radius. ${ }^{36}$

The usefulness of the above sum rules lies in that, under some conditions, one may obtain information about the dipole strength just from the ground state structure of the system. In particular, they allow to define two average excitation energies, ${ }^{32,33}$ namely, $E_{1} \equiv\left(m_{1} / m_{-1}\right)^{1 / 2}$ and $E_{3} \equiv\left(m_{3} / m_{1}\right)^{1 / 2}$, which give more weight either to the low-energy or to the high-energy part of the spectrum, respectively. If the excitation spectrum is concentrated in a fairly narrow energy region, $E_{1}$ or $E_{3}$ represent the mean energy of the excited mode. However, if the excitation spectrum is fairly fragmented, these mean energies can hardly represent the actual excitation energies. This is the present case, as we show in the next subsection.

\section{B. Real-time adiabatic time-dependent local spin-density theory}

We have calculated the density dipole response of the system using the real-time adiabatic TDLSDFT approach described in detail in Ref. 37, which we have restricted to our simpler 2D quantum ring. The analysis of the modes has been done following the method proposed in Ref. 38. Essentially, the procedure consists in considering the interaction with the dipole field as a small perturbation to the gs $\left|\Psi_{0}\right\rangle$ of the $N$-electron $\mathrm{QR}$ along a certain direction $\hat{\mathbf{e}}$, i.e., $\left|\Psi_{0, \hat{\mathbf{e}}}^{\prime}\right\rangle$ $\equiv e^{i \lambda \hat{\mathbf{e}} \mathbf{r}}\left|\Psi_{0}\right\rangle$, with $\lambda \ll 1$. To first order in $\lambda$, this can be written as $\left|\Psi_{0, \hat{\mathbf{e}}}^{\prime}\right\rangle \approx(1+i \lambda \hat{\mathbf{e}} \cdot \mathbf{r})\left|\Psi_{0}\right\rangle$. The ground and excited states of the Hamiltonian $\mathcal{H}$ constitute a basis $\left\{\left|\Psi_{0}\right\rangle,\left|\Psi_{j}\right\rangle\right\}(j \neq 0)$ in which the time evolution of the perturbed state can be expanded: $\quad\left|\Psi_{\hat{\mathbf{e}}}^{\prime}(t)\right\rangle=e^{-i \mathcal{H} t}\left|\Psi_{0, \hat{\mathrm{e}}}^{\prime}\right\rangle$ $\approx e^{-i \omega_{0} t}\left|\Psi_{0}\right\rangle+\sum_{j=1}^{\infty} a_{j, \hat{\mathbf{e}}} \hat{e}^{-i \omega_{j} t}\left|\Psi_{j}\right\rangle$. One can show that the quantity $\left\langle\Psi_{\hat{\mathbf{e}}}^{\prime}(t)|\hat{\mathbf{e}} \cdot \mathbf{r}| \Psi_{\hat{\mathbf{e}}}^{\prime}(t)\right\rangle-\left\langle\Psi_{0}|\hat{\mathbf{e}} \cdot \mathbf{r}| \Psi_{0}\right\rangle \equiv d_{\hat{\mathbf{e}}}(t)$ is related to the dipole strength function by the expression

$$
S_{\hat{\mathbf{e}}}(\omega)=\frac{1}{\pi \lambda} \int_{0}^{\infty} d_{\hat{\mathbf{e}}}(t) \sin \omega t d t=\sum_{j=1}^{\infty}\left|\left\langle\Psi_{j}|\hat{\mathbf{e}} \cdot \mathbf{r}| \Psi_{0}\right\rangle\right|^{2} \delta\left(\omega_{j 0}-\omega\right),
$$

where, as in the previous subsection, $\omega_{j 0} \equiv \omega_{j}-\omega_{0}$ is the excitation energy of the state $\left|\Psi_{j}\right\rangle$. Finally, to obtain the response frequencies of the system, we perform a least squares minimization of the time-discretized function defined as $\Sigma_{t}\left[d_{\hat{\mathbf{e}}}(t)-D_{\hat{\mathbf{e}}}(t)\right]^{2}$, with $D_{\hat{\mathbf{e}}}(t)$ given by

$$
D_{\hat{\mathbf{e}}}(t)=\sum_{j=1}^{M}\left[A_{j, \hat{\mathbf{e}}} \cos \omega_{j 0} t+B_{j, \hat{\mathbf{e}}} \sin \omega_{j 0} t\right],
$$

where the sum extends over $M$ frequencies of the system, $M$ being large enough to provide an accurate $d_{\hat{\mathbf{e}}}(t)$ from $D_{\hat{\mathbf{e}}}(t)$ and to assure the convergence of the calculation. The set $\left\{\omega_{j 0}\right\}$ is obtained from Eqs. (8) and (9) as a discrete set of Dirac delta functions that in practice we have smoothed out into fairly narrow Lorentzians.

As an example, Fig. 6 shows the dipole strength function corresponding to the $N=6$ ring for $B=5 \mathrm{~T}$ and $\mathcal{E}=0$ $-3 \mathrm{mV} / \mathrm{nm}$. This ring presents a change in the spin gs induced by the applied electric field, see Fig. 4. The dipole strength represents the sum of the contributions corresponding to the $\hat{\mathbf{x}}$ and $\hat{\mathbf{y}}$ directions, i.e., $S(\omega) \equiv S_{\hat{\mathbf{x}}}(\omega)+S_{\hat{\mathbf{y}}}(\omega)$. The more salient feature of the density dipole response when an $\mathcal{E}$ field is applied to the $\mathrm{QR}$ is its robustness. The $\mathcal{E}=0$ reference spectrum (bottom panel) shows a two peak structure around $\omega=5 \mathrm{meV}$ due to splitting caused by the magnetic field and some strength around $\omega=20 \mathrm{meV}$. This highenergy structure has been discussed in detail in Ref. 39 and 


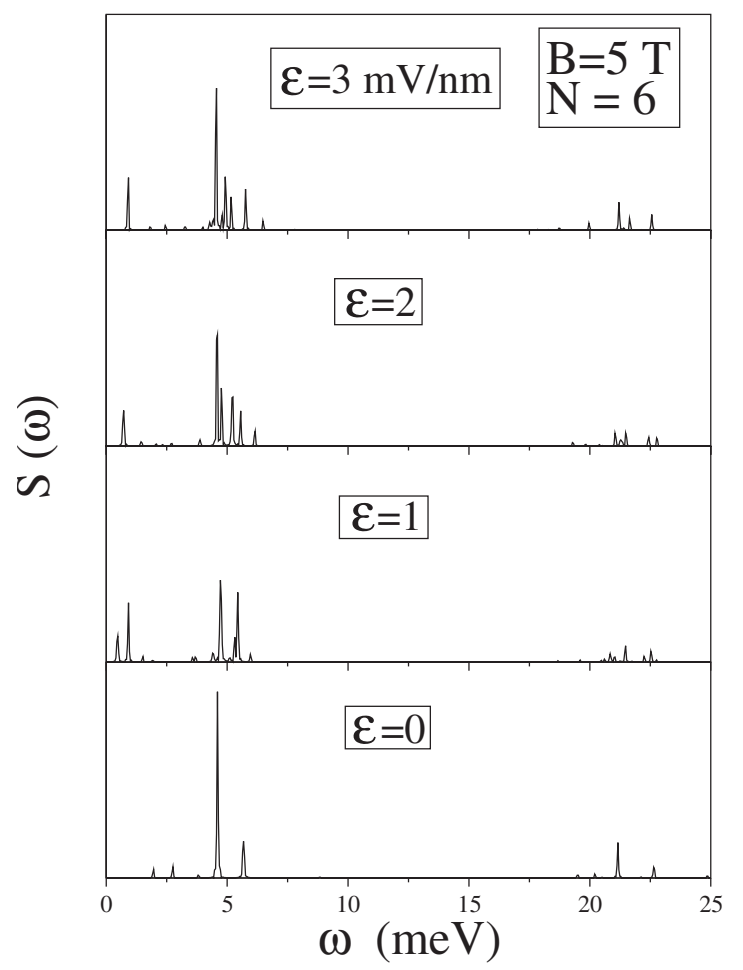

FIG. 6. Dipole strength function $S(\omega)$ (arbitrary units) as a function of the excitation energy $\omega$ corresponding to $N=6, B=5 \mathrm{~T}$, and several values of the electric field $\mathcal{E}$.

references therein and constitutes a signature of the QR geometry that shows up in its far-infrared spectrum. The upper panels show that both structures are clearly visible as $\mathcal{E}$ increases, with the only noticeable change that the dipole strength becomes more fragmented, and the appearance of a soft mode around $\omega=1 \mathrm{meV}$, which is absent when the system is axially symmetric (i.e., when $\mathcal{E}=0$ ).

\section{EXACT SOLUTION FOR THE TWO-ELECTRON QUANTUM RING}

For the two-electron system we have solved the Schrödinger equation with the Hamiltonian [Eq. (4)] using the method of Ref. 40, consisting in a uniform discretization of the $x y$ plane and using finite differences to evaluate the Laplacian for the kinetic energy. Associating an index with the positions of the two electrons $\left(\mathbf{r}_{1}, \mathbf{r}_{2}\right) \equiv I$, the resulting matrix equation reads $H_{I J} \Psi_{J}=E \Psi_{I}$. The Hamiltonian matrix is very sparse since only the kinetic term yields nondiagonal contributions to $H_{I J}$, being the external fields (ring potential and electric and magnetic fields), as well as the Coulomb interaction, local in $\left(\mathbf{r}_{1}, \mathbf{r}_{2}\right)$. The eigenvalue matrix equation can be solved by using iterative methods for boundary value problems. ${ }^{41}$ This way, one determines $E$ and $\Psi_{I}$ by repeated action of $H_{I J}$ on an arbitrary initial guess for $\Psi_{I}$. For the singlet (triplet) state, the wave function $\Psi\left(\mathbf{r}_{1}, \mathbf{r}_{2}\right)$ is symmetric (antisymmetric) with respect to the exchange of $\mathbf{r}_{1}$ and $\mathbf{r}_{2}$.

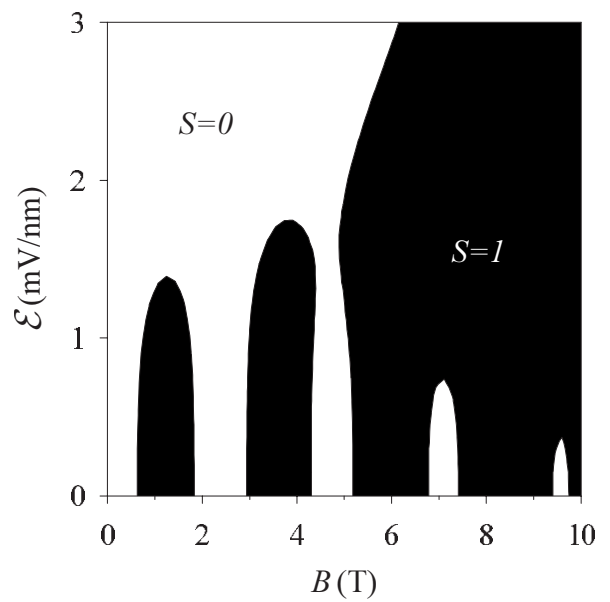

FIG. 7. Spin-phase diagram for the two-electron ring in the electric-magnetic field plane. Black (white) indicates a triplet (singlet) ground state.

This result implies that the triplet state vanishes for $I$ $=(\mathbf{r}, \mathbf{r})$, while the singlet has a cusp at these $I$ 's that compensates the divergence in the Coulomb interaction. To avoid this singularity, we do not impose the Schrödinger equation at these specific values for $I$, but directly enforce the zero value of $\Psi$ for the triplet and the cusp behavior for the singlet state. In order to extract the cusp condition, we extrapolate the wave function at the closer I's using the analytically known behavior for a parabolic confinement. ${ }^{42}$ Therefore, this method is reliable for smooth potentials that can be approximated by parabolas at a local scale. We have systematically checked the stability of the results by using finer grids in the calculations.

A detailed exploration of the $\mathcal{E}-B$ plane is presented in Fig. 7 with the spin-phase diagram corresponding to the ground state of the ring. The exact calculation shows the existence of spin islands at relatively low electric and magnetic fields that cause spin oscillations when, for a fixed $\mathcal{E}$, one increases $B$. The LSDFT calculations for the same $N$ $=2$ system (not shown here) also predict the possibility to induce singlet-triplet transitions by varying the intensity of the electric field at fixed magnetic field. However, we want to point out that, as expected, the LSDFT mean-field approach is unable to reproduce the details of the exact calculation, missing, in particular, the existence of spin islands and the associated spin oscillations.

\section{SUMMARY}

Within the local spin-density functional theory, we have calculated the addition energies and the density dipole excitation spectrum for quantum rings containing up to $N=12$ electrons, under the influence of both electric and magnetic fields. Transitions between states with spin $S=0$ and $S=1$ induced by $\mathcal{E}$ at fixed $B$ are found for some even values of $N$. For the two-electron ring, we have presented an exact calculation of the spin-phase diagram in the electric-magnetic field plane that, like the mean-field calculation, shows the possibility to induce singlet-triplet or triplet-singlet transi- 
tions by varying $\mathcal{E}$ and $B$. These spin transitions have been found in Coulomb blockade experiments with quantum dots and rings. The density dipole spectrum turns out to be very robust under the application of the electric field and shows the characteristic structure of quantum rings, with a $20 \%-$ $30 \%$ amount of the dipole strength appearing at fairly highenergy frequencies.

\section{ACKNOWLEDGMENTS}

This work has been performed under Grants No. FIS2005-01414 and No. FIS2005-02796 from DGI (Spain), No. 2005SGR00343 from Generalitat de Catalunya, and Grant No. INFN07-30 from the Italian INFN-Spanish DGI agreement.
${ }^{1}$ S. Lüscher, T. Heinzel, K. Ensslin, W. Wegscheider, and M. Bichler, Phys. Rev. Lett. 86, 2118 (2001).

${ }^{2}$ T. Ihn, A. Fuhrer, T. Heinzel, K. Ensslin, W. Wegscheider, and M. Bichler, Physica E (Amsterdam) 16, 83 (2003).

${ }^{3}$ D. S. Duncan, D. Goldhaber-Gordon, R. M. Westervelt, K. D. Maranowski, and A. C. Gossard, Appl. Phys. Lett. 77, 2183 (2000).

${ }^{4}$ J. A. Folk, C. M. Marcus, R. Berkovits, I. L. Kurland, I. L. Aleiner, and B. L. Altshuler, Phys. Scr., T T90, 26 (2001).

${ }^{5}$ S. Lindemann, T. Ihn, T. Heinzel, W. Zwerger, K. Ensslin, K. D. Maranowski, and A. C. Gossard, Phys. Rev. B 66, 195314 (2002).

${ }^{6}$ R. Hanson, B. Witkamp, L. M. K. Vandersypen, L. H. Willems van Beveren, J. M. Elzerman, and L. P. Kouwenhoven, Phys. Rev. Lett. 91, 196802 (2003).

${ }^{7}$ L. P. Rokhinson, L. J. Guo, S. Y. Chou, and D. C. Tsui, Phys. Rev. B 63, 035321 (2001).

${ }^{8}$ M. Ciorga, A. Wensauer, M. Pioro-Ladriere, M. Korkusinski, J. Kyriakidis, A. S. Sachrajda, and P. Hawrylak, Phys. Rev. Lett. 88, 256804 (2002).

${ }^{9}$ K. Ono, D. G. Austing, Y. Tokura, and S. Tarucha, Science 297, 1313 (2002).

${ }^{10}$ A. K. Hüttel, H. Quin, A. W. Holleitner, R. H. Blick, K. Neumaier, D. Weinmann, K. Eberl, and J. P. Kotthaus, Europhys. Lett. 62, 712 (2003).

${ }^{11}$ R. C. Ashoori, H. L. Stormer, J. S. Weiner, L. N. Pfeiffer, K. W. Baldwin, and K. W. West, Phys. Rev. Lett. 71, 613 (1993).

${ }^{12}$ T. Schmidt, M. Tewordt, R. H. Blick, R. J. Haug, D. Pfannkuche, K. v. Klitzing, A. Förster, and H. Lüth, Phys. Rev. B 51, 5570 (1995).

${ }^{13}$ L. P. Kouwenhoven, T. H. Oosterkamp, M. W. S. Danoesastro, M. Eto, D. G. Austing, T. Honda, and S. Tarucha, Science 278, 1788 (1997).

${ }^{14}$ S. Tarucha, D. G. Austing, Y. Tokura, W. G. van der Wiel, and L. P. Kouwenhoven, Phys. Rev. Lett. 84, 2485 (2000).

${ }^{15}$ M. Ciorga, A. S. Sachrajda, P. Hawrylak, C. Gould, P. Zawadzki, Y. Feng, and Z. Wasilewski, Physica E (Amsterdam) 11, 35 (2001).

${ }^{16}$ M. Ciorga, A. S. Sachrajda, and P. Hawrylak, J. Supercond. 16, 743 (2003).

${ }^{17}$ G. Burkard, D. Loss, and D. P. DiVincenzo, Phys. Rev. B 59, 2070 (1999).

${ }^{18}$ X. Hu and S. DasSarma, Phys. Rev. A 61, 062301 (2000).
${ }^{19}$ G. Burkard, G. Seelig, and D. Loss, Phys. Rev. B 62, 2581 (2000).

${ }^{20}$ D. Loss and D. P. DiVincenzo, Phys. Rev. A 57, 120 (1998).

${ }^{21}$ J. Kyriakidis, M. Pioro-Ladriere, M. Ciorga, A. S. Sachrajda, and P. Hawrylak, Phys. Rev. B 66, 035320 (2002).

${ }^{22}$ A. Fuhrer, T. Ihn, K. Ensslin, W. Wegscheider, and M. Bichler, Phys. Rev. Lett. 91, 206802 (2003).

${ }^{23}$ T. Ihn, A. Fuhrer, K. Ensslin, W. Wegscheider, and M. Bichler, Physica E (Amsterdam) 26, 225 (2005).

${ }^{24}$ A. Fuhrer, S. Lüscher, T. Ihn, T. Heinzel, K. Ensslin, W. Wegscheider, and M. Bichler, Nature (London) 413, 822 (2001).

${ }^{25}$ B. Tanatar and D. M. Ceperley, Phys. Rev. B 39, 5005 (1989).

${ }^{26}$ U. von Barth and L. Hedin, J. Phys. C 5, 1629 (1972).

${ }^{27}$ M. Frigo and S. G. Johnson, Proc. IEEE 93, 216 (2005).

${ }^{28}$ F. Ancilotto, D. G. Austing, M. Barranco, R. Mayol, K. Muraki, M. Pi, S. Sasaki, and S. Tarucha, Phys. Rev. B 67, 205311 (2003).

${ }^{29}$ S. M. Reimann and M. Manninen, Rev. Mod. Phys. 74, 1283 (2002).

${ }^{30}$ A. Emperador, M. Pi, M. Barranco, and E. Lipparini, Phys. Rev. B 64, 155304 (2001).

${ }^{31}$ J. C. Lin and G. Y. Guo, Phys. Rev. B 65, 035304 (2001).

${ }^{32}$ O. Bohigas, A. M. Lane, and J. Martorell, Phys. Rep. 51, 267 (1979).

${ }^{33}$ E. Lipparini and S. Stringari, Phys. Rep. 175, 103 (1989).

${ }^{34}$ E. Lipparini, Modern Many Particle Physics-Atomic Gases, Quantum Dots and Quantum Fluids (World Scientific, Singapore, 2003).

${ }^{35} \mathrm{M}$. Weissbluth, Atoms and Molecules (Academic, New York, 1978).

${ }^{36}$ Ll. Serra, F. Garcias, M. Barranco, J. Navarro, C. Balbás, A. Rubio, and A. Mañanes, J. Phys.: Condens. Matter 1, 10391 (1989).

${ }^{37}$ M. Pi, F. Ancilotto, E. Lipparini, and R. Mayol, Physica E (Amsterdam) 24, 297 (2004).

${ }^{38}$ A. Puente and Ll. Serra, Phys. Rev. Lett. 83, 3266 (1999).

${ }^{39}$ J. I. Climente, J. Planelles, and F. Rajadell, J. Phys.: Condens. Matter 17, 1573 (2005).

${ }^{40}$ A. Puente and Ll. Serra, Phys. Rev. B 63, 125334 (2001).

${ }^{41}$ S. E. Koonin and D. C. Meredith, Computational Physics (Addison-Wesley, Reading, MA, 1990).

${ }^{42}$ J.-L. Zhu, J.-Z. Yu, Z.-Q. Li, and Y. Kawazoe, J. Phys.: Condens. Matter 8, 7857 (1996). 